\title{
Frame Theory and Optimal Anchor Geometries in Wireless Localization
}

\author{
Samuel Van de Velde, Student Member, IEEE, Giuseppe Abreu, Senior Member, IEEE, \\ and Heidi Steendam, Senior Member, IEEE
}

\begin{abstract}
We revisit the problem of describing optimal anchor geometries that result in the minimum achievable MSE by employing the Cramer Rao Lower bound. Our main contribution is to show that this problem can be cast onto the whelm of modern Frame Theory, which not only provides new insights, but also allows the straightforward generalization of various classical results for the anchor placement problem. For example, by employing the frame potential for single-target localization, we see that the directions of the anchors, as seen from the target, should optimally be as orthogonal as possible, and that the existence of an optimal geometry for an arbitrary number of anchors is governed by the fundamental inequality in frame theory. Furthermore, the frame-theoretic approach allows for the simple derivation of some properties on optimal anchor placement that prove to be useful in a tractable approach for the more complex, multi-target anchor placement problem.

In a more general sense, the paper builds a refreshing bridge between the classical problem of wireless localization and the powerful domain of Frame Theory, with far-reaching potential.
\end{abstract}

Index Terms-Localization, anchor placement, tight frame

\section{INTRODUCTION}

In wireless localization, the user estimates its position by making a set of measurements with a number of fixed reference points called anchors. It is known that the geometry of these anchors with respect to the user can have a tremendous impact on the accuracy of the position estimate [1]. By studying the Cramer Rao Lower Bound (CRLB), which bounds the mean squared error of an efficient estimator, it is possible to understand the role that the anchor geometry plays in the localization problem. From such an analytical study it becomes possible to obtain so called optimal geometries that minimize the CRLB, or to identify the importance of each individual anchor on localization.

When a localization system is deployed from scratch, some results on where to place the set of anchors, with respect to a single target, are already known. In [2], [3], [4], it was shown that a uniform perimeter placement is optimal in 2D. In [2], the authors suggest that anchors "equally" distributed on a unit spherical surface provide optimal geometries. In 3D, this results in geometries where anchors are placed on the vertices of one of the five platonic solids. However, these solutions only provide geometries for a specific number of anchors and it was not known if an optimal geometry exists for any number of anchors. Also, these results are obtained under the condition that all range-measurements have an identical noise model.

In this paper, we will show that the optimal anchor placement is in fact related to frame theory [5]. Frame theory is

S. Van de Velde and H. Steendam are with the Department of Telecommunications and Information Processing TELIN, Ghent University, Belgium, e-mail: $\{$ slvdveld, hs $\} @$ telin.ugent.be.

G. Abreu is with the School of Engineering and Science, Jacobs University Bremen gGmbH, Campus Ring 1, 28759 Bremen, Germany (e-mail: g.abreu@jacobs-university.de). a generalization of bases and has gained much popularity the last decade. In contrast to a basis, where the vectors in the set must span the vector space and must be linearly independent, a frame is a generalization of a basis in the sense that the set of vectors contains more vectors than needed to span the vector space, and as a result, the vectors in the set will be linearly dependent. Hence, a frame can be seen as the redundant counterpart of a basis and because of this, they have been successfully applied in fields where redundancy is the key such as coding theory, robust transmission, CDMA systems, etc [6]. In wireless localization there always exists a minimum number of anchors needed for localization. Consequently, when more anchors are used than necessary some kind of redundancy is introduced into the system and we can intuitively understand that a connection with frame theory exists. From frame theory we can obtain conditions on the geometries of these redundant sets of anchors such that optimality is achieved.

We show that these results generalize previously known results on the optimal anchor placement, while contributing to the intuitive understanding of the problem. Furthermore, by rigorously solving the single-target problem, we also gain understanding in the optimal geometry for multi-target localization. As a result of this analysis, we propose a lowcomplexity algorithm for sequential anchor deployment in large networks.

\section{Problem Statement}

Consider a wireless network with $N$ target nodes that are to be localized and $K$ anchor nodes with known positions. The node coordinates are denoted by column vectors $\mathbf{x}_{i} \in \mathbb{R}^{M}$ ( $M=2,3$ for 2D and 3D localization, respectively) where for targets $i \in\{1, . ., N\}$ and for anchors $i \in\{N+1, . ., N+K\}$. Every node $i$ is connected to a number of neighboring nodes (both targets and anchors), collected in the set $\mathcal{N}(i)$, with whom ranging can be performed. The noisy range measurement between node $i$ and $j$ is modelled as follows:

$$
r_{i j}=\left\|\mathbf{x}_{i}-\mathbf{x}_{j}\right\|+n_{i j}
$$

where $n_{i j}$ is a noise-term that is described by the probability density function (PDF) $p_{i j}\left(n_{i j}\right)$ where we assume that the PDFs satisfy the Cramèr-Rao regularity conditions [7].

Consider the estimation of all target coordinates, aggregated in a vector $\boldsymbol{\theta}=\left[\mathbf{x}_{1}^{\mathrm{T}}, \mathbf{x}_{2}^{\mathrm{T}}, \ldots, \mathbf{x}_{N}^{\mathrm{T}}\right]^{\mathrm{T}}$. It is well-known [7] that the covariance matrix of an unbiased estimator $\hat{\boldsymbol{\theta}}$ is bounded by the CRLB

$$
\mathbb{E}_{\hat{\boldsymbol{\theta}}}\left[(\boldsymbol{\theta}-\hat{\boldsymbol{\theta}})(\boldsymbol{\theta}-\hat{\boldsymbol{\theta}})^{\mathrm{T}}\right] \succeq \mathbf{F}^{-1},
$$

where $\mathbb{E}_{\hat{\theta}}[\cdot]$ denotes the expectation over the distribution of $\hat{\boldsymbol{\theta}}$, the symbol $\succeq$ denotes positive-semidefinite inequality and $\mathbf{F}^{-1}$ is the pseudo inverse of the Fisher information matrix. 
Consequently, the mean squared error (MSE) of the position estimate is bounded by trace $\left(\mathbf{F}^{-1}\right)$. In this paper we will use this bound as a metric for optimization.

\section{OPTIMAL GEOMETRY AND TIGHT FRAMES}

In this section we address the problem of optimally placing all $K$ anchors for a single target, i.e., $N=1$. Before we present our main results, let us introduce a definition that will be used in the Theorem presented immediately after.

Definition 1 (Frame and Tight Frame).

A finite sequence of vectors $\left\{\mathbf{e}_{k}\right\}_{k=1}^{K}$ in $\mathbb{R}^{M}$, for $K \geq M$, is called a frame if there exists two scalars $A, B>0$ such that the following condition is satisfied for any $\mathbf{y} \in \mathbb{R}^{M}$ :

$$
A\|\mathbf{y}\|^{2} \leq \sum_{k=1}^{K}\left|\mathbf{y}^{\mathrm{T}} \mathbf{e}_{k}\right|^{2} \leq B\|\mathbf{y}\|^{2}
$$

where the scalars, $A$ and $B$ are known as the frame bounds.

Tight frames are a class of frames where $A=B$. When all vectors $\left\{\mathbf{e}_{k}\right\}_{k=1}^{K}$ have an equal norm we call the frame an equal-norm frame.

Theorem 1 (Optimal Anchor Placement).

A set of $K$ anchors form an optimal geometry for range-based localization when the range vectors $\mathbf{u}_{k}$, defined by:

$$
\mathbf{u}_{k} \triangleq \sqrt{F_{k}} \frac{\mathbf{x}_{1}-\mathbf{x}_{1+k}}{\left\|\mathbf{x}_{1}-\mathbf{x}_{1+k}\right\|}
$$

where $F_{k}$ is the Fisher information associated with $p_{1,1+k}\left(n_{1,1+k}\right)$, form a tight frame, i.e.,

$$
\sum_{k=1}^{K}\left|\mathbf{y}^{\mathrm{T}} \mathbf{u}_{k}\right|^{2}=\lambda\|\mathbf{y}\|^{2}
$$

Proof: First, we show that, to obtain optimality in the sense of minimizing trace $\left(\mathbf{F}^{-1}\right)$, the eigenvalues of the FIM $\mathbf{F}$ must be equal, i.e $\mathbf{F}=\lambda \mathbf{I}_{M}$. Let us consider the eigendecomposition of the symmetric, positive semidefinite FIM $\mathbf{F}=\mathbf{Q} \mathbf{\Lambda} \mathbf{Q}^{\mathrm{T}}$. Hence, the eigenvalues of $\mathbf{F}$ will be real-valued and non-negative. Since $\mathbf{Q}^{\mathrm{T}}=\mathbf{Q}^{-1}$, the CRLB can be written as

$\operatorname{trace}\left(\mathbf{F}^{-1}\right)=\operatorname{trace}\left(\mathbf{Q}^{\mathrm{T}} \boldsymbol{\Lambda}^{-1} \mathbf{Q}\right)=\operatorname{trace}\left(\boldsymbol{\Lambda}^{-1}\right)=\sum_{m=1}^{M} \frac{1}{\lambda_{m}}$.

It is trivial to show that this expression is minimal if and only if $\lambda_{m}=\lambda$ for $\forall m$, which in turn implies that optimality is achieved iff $\mathbf{F}=\lambda \mathbf{I}_{M}$.

The Fisher Information Matrix $\mathbf{F}$ of the parameter vector $\mathbf{x}$ can easily be obtained by noting that the set of measurements as described in (1) are simply a parameter transformation of $\mathbf{x}$. Because of this we can use the following formula for the FIM [7]:

$$
\mathbf{F}=\mathbf{J}^{\mathrm{T}} \mathbf{F}_{r} \mathbf{J}
$$

with $\mathbf{J}$ the $K \times M$ Jacobian matrix where the $k$ th row is given by

$$
\mathbf{s}_{k}=\frac{\partial\left\|\mathbf{x}_{1}-\mathbf{x}_{1+k}\right\|}{\partial \mathbf{x}_{1}^{\mathrm{T}}}=\frac{\left(\mathbf{x}_{1}-\mathbf{x}_{1+k}\right)^{\mathrm{T}}}{\left\|\mathbf{x}_{1}-\mathbf{x}_{1+k}\right\|}
$$

and $\mathbf{F}_{r}$ the $K \times K$ Fisher Information Matrix of the inputs $\left\{r_{1,1+k}\right\}_{k=1}^{K}$. Because we assume all measurements to be independent, $\mathbf{F}_{k}$ is a diagonal matrix where the $k$ th diagonal element is the scalar Fisher information $F_{k}=$ $\mathbb{E}\left[\frac{\partial \log p_{1,1+k}\left(n_{1,1+k}\right)}{\partial n_{1,1+k}}\right]$ corresponding to the range measurement with the $k$ th anchor. Given the definition of the range vectors in (4), we can write the FIM as

$$
\mathbf{F}=\sum_{k=1}^{K} \mathbf{u}_{k} \mathbf{u}_{k}^{\mathrm{T}}
$$

This results in the following condition for optimality:

$$
\sum_{k=1}^{K} \mathbf{u}_{k} \mathbf{u}_{k}^{\mathrm{T}}=\lambda \mathbf{I}_{M}
$$

It is left for us to show that equation (10) in fact implies that the set of real-valued information vectors $\left\{\mathbf{u}_{k}\right\}_{k=1}^{K}$ is a tight frame. To this end, multiply equation (10) to the right by $\mathbf{y} \in \mathbb{R}^{M}$ and to the left by $\mathbf{y}^{\mathrm{T}}$, such that

$$
\begin{aligned}
\mathbf{y}^{\mathrm{T}}\left(\sum_{k=1}^{K} \mathbf{u}_{k} \mathbf{u}_{k}^{\mathrm{T}}\right) \mathbf{y} & =\mathbf{y}^{\mathrm{T}} \lambda \mathbf{I}_{M} \mathbf{y}, \\
\sum_{k=1}^{K}\left(\mathbf{y}^{\mathrm{T}} \mathbf{u}_{k}\right)\left(\mathbf{u}_{k}^{\mathrm{T}} \mathbf{y}\right) & =\lambda \mathbf{y}^{\mathrm{T}} \mathbf{y}, \\
\sum_{k=1}^{K}\left(\mathbf{y}^{\mathrm{T}} \mathbf{u}_{k}\right)\left(\mathbf{y}^{\mathrm{T}} \mathbf{u}_{k}\right) & =\lambda \mathbf{y}^{\mathrm{T}} \mathbf{y}, \\
\sum_{k=1}^{K}\left|\mathbf{y}^{\mathrm{T}} \mathbf{u}_{k}\right|^{2} & =\lambda\|\mathbf{y}\|^{2},
\end{aligned}
$$

which defines a tight frame, as per Definition 1, thus concluding the proof.

The beauty of Theorem 1 is that it casts the seemingly intractable problem of optimal anchor geometries onto the rich mathematical framework of Finite Frame Theory [8], lending the problem a vast number of analytical tools and results which can now be applied not only to solve the optimization problem, but also to understand it. For this, it is important to note that the range vectors $\mathbf{u}_{k}$ defined in Theorem 1 are simply $M$-dimensional vectors pointing towards their corresponding anchor; the norm of these range vectors is determined by the noise model of the range measurement. For example, if we consider normally distributed noise with zero mean and standard deviation $\sigma_{k}$ for the measurement $r_{1,1+k}$, then $F_{k}=\frac{1}{\sigma_{k}^{2}}$.

Before offering explicit geometries that provide optimality, important insight can be gained based on the known properties of tight frames. In the following, a selected list of such properties is given.

1) Minimal Inclusion: A tight frame $\left\{\mathbf{u}_{k}\right\}_{k=1}^{K}$ in $\mathbb{R}^{M}$ with $K=M$ is an orthogonal basis of $\mathbb{R}^{M}$.

2) Superposition: Let $\left\{\mathbf{u}_{k}\right\}_{k=1}^{K}$ and $\left\{\mathbf{v}_{\ell}\right\}_{\ell=1}^{L}$ be distinct tight frames in $\mathbb{R}^{M}$. Then, the superposition $\left\{\mathbf{u}_{k}, \mathbf{v}_{\ell}\right\}_{(k, \ell)}^{K, L}$ is also a tight frame in $\mathbb{R}^{M}$.

3) Sign Invariance: A tight frame remains tight after switching the sign of any frame vector $\mathbf{u}_{k}$.

4) Constructibility: For any dimension $M$ there exist tight sets with $K \geq M$ iff

$$
\max _{k \in\{1, \cdots, K\}}\left\|\mathbf{u}_{k}\right\|^{2} \leq \frac{1}{M} \sum_{k=1}^{K}\left\|\mathbf{u}_{k}\right\|^{2} .
$$


This condition is also known as the fundamental inequality.

5) Minimum Potential: Every frame has an associated frame potential,

$$
\varrho=\sum_{k, \ell}\left|\mathbf{u}_{k}^{\mathrm{T}} \mathbf{u}_{\ell}\right|^{2}
$$

which for a given set of norms of $\left\{\mathbf{u}_{k}\right\}_{k=1}^{K}$ is minimum when the frame is tight.

In light of Theorem 1, the above-listed properties have various important implications onto the construction of optimal anchor geometries. In fact, many of the known results on optimal geometries that have been derived in previous works [2], [4] are directly related to Properties 1 through 3 of tight frames.

More interestingly, some new insights can also be gained from the connection with tight frames. For instance, Property 4 implies that it is always possible to find an optimal geometry for both $2 \mathrm{D}$ and $3 \mathrm{D}$ localization, as long as the norms of the range vectors satisfy the fundamental inequality. When the noise models for the range measurements are identical, this immediately shows that optimal geometries exist for any number of anchors. This is a generalization of the result in [2] where only a few optimal geometries in 3D are provided, i.e. the five platonic solids and superpositions of those. Furthermore, Property 5 tells us that an optimal placement of $K$ anchors minimizes the potential $\mathrm{FP}=\sum_{k, \ell}\left|\mathbf{u}_{k}^{\mathrm{T}} \mathbf{u}_{\ell}\right|^{2}$. This frame potential is the result of forces that are assigned to each vector (and thus anchor).

In [8], it was found that this force was not a force found in nature but a so-called "orthogonality promoting" force. In contrast, in [2], it was suggested that the anchors should be "equally" distributed on the unit spherical surface for optimality. Although this happens to be true for the platonic solids, this equal distribution is in fact not a necessary condition. We can state the following:

1) Anchors should be placed as orthogonal as possible to obtain an optimal geometry.

2) Anchors that provide more accurate range measurements, resulting in a larger ranging vector, should be placed more orthogonal to the remaining anchors.

This last statement can be understood directly by inspecting the formula for the frame potential and the fundamental inequality for tight frames.

Consider now the following corollary of Theorem 1.

\section{Corollary 1 (CRLB of Optimum Estimator).}

Let $K \geq M$, and consider an optimum unbiased estimation of $\mathbf{x}$ given a set of range vectors $\left\{\mathbf{u}_{k}\right\}_{k=1}^{K}$ that form a tight frame. The CRLB associated with this estimator is

$$
\operatorname{trace}\left(\mathbf{F}^{-1}\right)=\frac{M^{2}}{\sum_{k=1}^{K} F_{k}} .
$$

Proof: Taking the trace of equation (9) yields

$$
\operatorname{trace}(\mathbf{F})=\operatorname{trace}\left(\sum_{k=1}^{K} \mathbf{u}_{k} \mathbf{u}_{k}^{\mathrm{T}}\right)=\sum_{k=1}^{K}\left\|\mathbf{u}_{k}\right\|^{2}=\sum_{k=1}^{K} F_{k} .
$$

On the other hand, from the optimality condition we have,

$$
\operatorname{trace}(\mathbf{F})=\operatorname{trace}\left(\lambda \mathbf{I}_{M}\right)=\lambda M .
$$
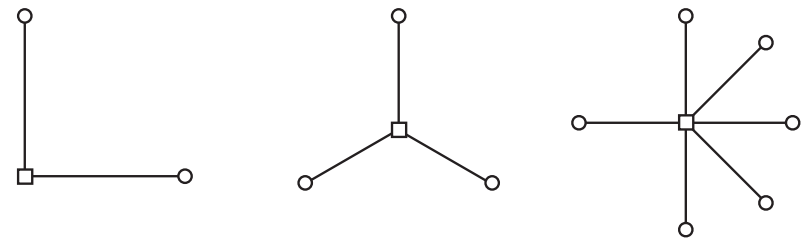

Fig. 1: Some example tight frames in 2D. From left to right: the orthogonal basis, the Mercedes Benz frame [5] and a superposition of a harmonic tight frame and the orthogonal basis. The circles indicate anchor positions and the squares represents the target.
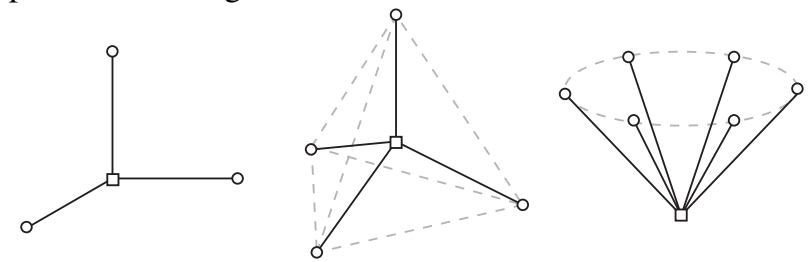

Fig. 2: Some example tight frames in 3D. From left to right: the orthogonal basis, the vertices of a tetrahedron and the harmonic frame. The circles indicate anchor positions and the squares represents the target.

Combining the latter equations we have $\lambda=\frac{1}{M} \sum_{k=1}^{K} F_{k}$, and consequently

$$
\operatorname{trace}\left(\mathbf{F}^{-1}\right)=\operatorname{trace}\left(\frac{1}{\lambda} \mathbf{I}_{M}\right)=\frac{M}{\lambda}=\frac{M^{2}}{\sum_{k=1}^{K} F_{k}},
$$

which concludes the proof.

Corollary 1 tells us that $\sum_{k=1}^{K} F_{k}$ will determine the CRLB as long as the corresponding range vectors form a tight frame. The $F_{k}$ 's are directly related to the noise distribution of the observations meaning that noise distributions do not need to be identical to achieve optimality. However, having all $F_{k}$ 's identical has the advantage that the range vectors all have an equal norm: it is easier to obtain and describe equal-norm tight frames. An example of an equal-norm tight frame is the Harmonic frame. For $\mathbb{R}^{2}$ and $\mathbb{R}^{3}$ the Harmonic frame vectors are given by:

$$
\mathbf{u}_{k}=\frac{2}{\sqrt{2}}[\cos (2 \pi k / K), \sin (2 \pi k / K)]
$$

and

$$
\mathbf{u}_{k}=\frac{2}{\sqrt{3}}\left[\cos (2 \pi k / K), \sin (2 \pi k / K), \frac{1}{\sqrt{2}}\right],
$$

respectively.

For $M=2$ it can be seen that this frame corresponds to the uniform perimeter placement of anchors as was also observed in [2], [3] and [4]. Other examples of equal-norm tight frames in 2D are shown in Fig. 1. In 3D, the harmonic frame gives optimal geometries for any number of anchors. With this specific frame, it is seen from Fig. 2 that it is possible to obtain good positioning accuracy with all anchors in a plane, elevated above the target. Other examples of equalnorm tight frames for $M=3$ are the vectors described by the vertices of platonic solids. As such the Tetrahedron, Hexahedron, Octahedron, Dodecahedron and Icosahedron thus describe optimal anchor geometries. This corresponds to the findings in [2]. Another example is the truncated icosahedron, also known as the soccerbal [8]. 


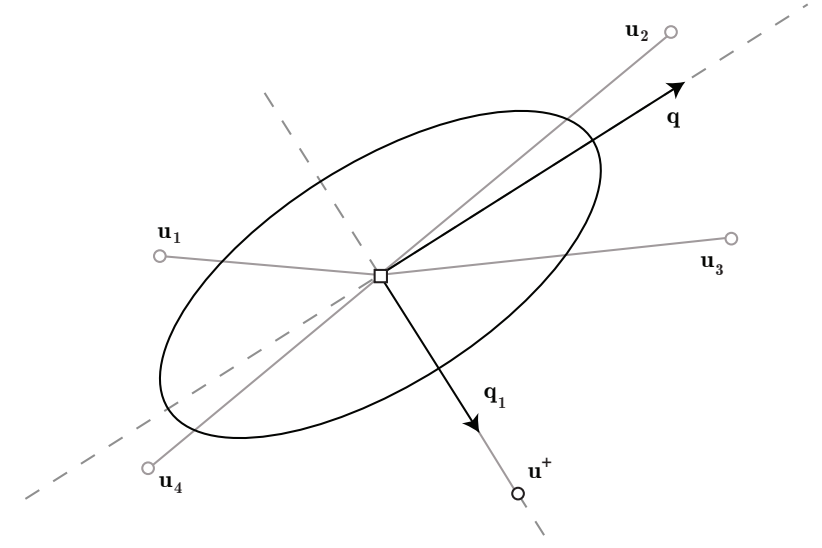

Fig. 3: Information ellipse of $\mathbf{F}$ given by $\mathbf{y} \mathbf{F}^{-1} \mathbf{y}=1$, for two-dimensional localization. The range vector $\mathbf{u}^{+}$of the new anchor $\mathrm{x}^{+}$(and thus the new anchor as well) is in the direction of the minor eigenvector $\mathbf{q}_{1}$ of $\mathbf{F}$.

Whenever the noise models for the anchors are not identical, tight frames can still be obtained such as described in [9], where a frame construction procedure, similar to the GramSchmidt method for orthogonal bases is proposed.

To conclude this section on single-target optimal geometries, we present the following Theorem that will prove to be useful in the following section where multi-target networks are considered.

\section{Theorem 2 (Optimal Network Augmentation).}

Consider an anchor geometry that consists of a set of $K$ range vectors $\mathbf{u}_{k}$. Associated is the Fisher Information matrix $\mathbf{F}$ with minor eigenvector $\mathbf{q}_{1}$, i.e., the eigenvector corresponding to the smallest eigenvalue. The CRLB associated with an augmented geometry, where a single anchor is added, is minimized iff the range vector of the new anchor $\mathbf{u}^{+}$is aligned with $\mathbf{q}_{1}$, i.e.

$$
\mathbf{u}^{+} \cdot \mathbf{q}_{1}= \pm\left\|\mathbf{u}^{+}\right\|\left\|\mathbf{q}_{1}\right\| .
$$

Proof: See Appendix.

A visual representation of the above theorem is shown in Fig. 3 for $2 \mathrm{D}$ where a fifth anchor is added to an original topology of four anchors.

\section{ANCHOR PLACEMENT IN MULTI-TARGET NETWORKS}

Next, let us address the problem of finding criteria for the optimal placement of anchors in a localization network with multiple (possibly cooperating) targets. For more details around cooperation in wireless networks we refer to [10]. Following a similar reasoning as in Theorem 1, we can write the Fisher information matrix associated with the estimation of $\boldsymbol{\theta}=\left[\mathbf{x}_{1}^{\mathrm{T}}, \mathbf{x}_{2}^{\mathrm{T}}, \ldots, \mathbf{x}_{N}^{\mathrm{T}}\right]^{\mathrm{T}}$ as a sum of vector products,

$$
\mathbf{F}=\sum_{i=1}^{N} \sum_{j \in \mathcal{N}_{j}} \mathbf{v}_{i j} \mathbf{v}_{i j}^{\mathrm{T}} .
$$

When $\mathbf{u}_{i j}=\sqrt{F_{i j}} \frac{\mathbf{x}_{i}-\mathbf{x}_{j}}{\left\|\mathbf{x}_{i}-\mathbf{x}_{j}\right\|}$, the vectors $\mathbf{v}_{i j}$ describing the FIM are given by

$\mathbf{v}_{i j}=\left[\mathbf{0}_{1 \times M \cdot(i-1)}, \mathbf{u}_{i j}^{\mathrm{T}}, \mathbf{0}_{1 \times M \cdot(j-i-1)},-\mathbf{u}_{i j}^{\mathrm{T}}, \mathbf{0}_{1 \times M \cdot(N-j)}\right]^{\mathrm{T}}$ whenever the corresponding range measurement $r_{i j}$ (1) is between two targets $(j \leq N)$, and

$$
\mathbf{v}_{i j}=\left[\mathbf{0}_{1 \times M \cdot(i-1)}, \mathbf{u}_{i j}^{\mathrm{T}}, \mathbf{0}_{1 \times M \cdot(N-i)}\right]^{\mathrm{T}},
$$

when the corresponding range measurement $r_{i j}$ is between a target and an anchor $(j>N)$.

Notice that, due of the construction of $\mathbf{F}$, Theorem 1 remains valid, i.e., optimality is achieved when the set of vectors $\mathbf{v}_{i j}$ form a tight frame. However, unlike the single-target case, we cannot freely choose every vector $\mathbf{v}_{i j}$ independently by changing the anchor positions. From equation (22) we see that the vectors are fixed when $j \leq N$, and from equation (23), we see that changing one anchor position may affect multiple vectors $\mathbf{v}_{i j}$ when $j>N$. Because of this it becomes prohibitively challenging to find a tight frame for this problem. In fact, it may even not be possible to find a tight frame for any arbitrary network configuration. As a result we are forced to turn to an algorithmic approach. However, simply calculating the CRLB by taking the trace of the inverse of (21) for every anchor configuration, quickly becomes computationally intractable for larger networks. As an alternative, we propose an approach where anchors are sequentially added to the network.

\section{A. A Frame potential approach}

From Theorem 2, we know how to optimally place a new anchor for a single target, by minimizing the frame potential. We now extend this to the multi-target case by placing the new anchor "as much as possible" in the preferred direction of each target (see Fig. 4 for a graphical representation of this approach). Notice that this new problem is closely related to the problem of single-target localization using angle-of-arrival (AoA) measurements. In [11], a low-complex algorithm is proposed for solving the AoA problem. Here we will build upon the algorithm in [11] and additionally introduce weights that penalize deviations from the preferred direction more heavily, whenever that direction is more pronounced (i.e. when the FIM ellipse is narrow).

Consider the two-dimensional case $M=2$ where the position of the new anchor is denoted by $\mathbf{x}^{+}=\left[x^{+}, y^{+}\right]^{\mathrm{T}}$. The optimal direction of the new anchor, with respect to the $i$ th target is described by the angle $\alpha_{i}$ and can be obtained by calculating the FIM of the network in its current state (without the new anchor). We can impose the following relation:

$$
\tan \left(\alpha_{i}\right)=\frac{y^{+}-y_{i}}{x^{+}-x_{i}}
$$

For the $N$ different targets we can define $N$ such equations resulting in the linear system $\mathbf{A x}^{+}=\mathbf{b}$ with

$$
\mathbf{A}=\left[\begin{array}{cc}
1 & -\tan \left(\alpha_{1}\right) \\
1 & -\tan \left(\alpha_{2}\right) \\
\vdots & \vdots \\
1 & -\tan \left(\alpha_{N}\right)
\end{array}\right] \text {, and } \mathbf{b}=\left[\begin{array}{c}
y_{1}-x_{1} \tan \left(\alpha_{1}\right) \\
y_{2}-x_{2} \tan \left(\alpha_{2}\right) \\
\vdots \\
y_{N}-x_{N} \tan \left(\alpha_{N}\right)
\end{array}\right]
$$

For $N>2$ it is no longer possible to satisfy every condition and therefore we introduce a weighted least squares (WLS) objective function: $\left(\mathbf{A x} \mathbf{x}^{+}-\mathbf{b}\right)^{\mathrm{T}} \mathbf{W}\left(\mathbf{A x}^{+}-\mathbf{b}\right)$ with the elements of the diagonal matrix $\mathbf{W}$ given by

$$
W_{i i}=\left(\lambda_{i}^{\max }-\lambda_{i}^{\min }\right)^{2}\left(1+\left|\tan \left(\alpha_{i}\right)\right|\right)^{-2} .
$$




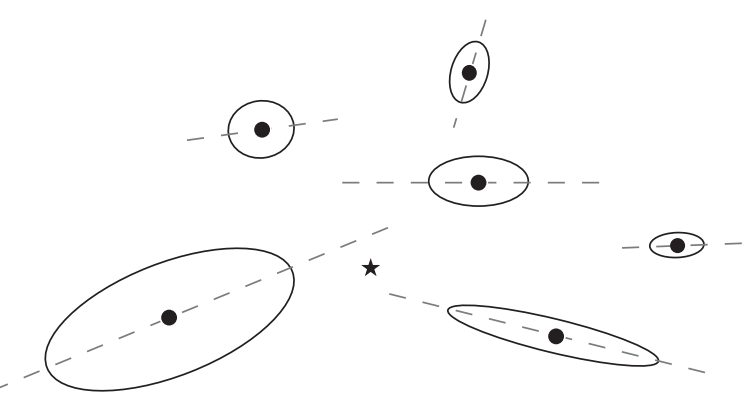

Fig. 4: A multi-target network where the uncertainty ellipses for each target indicate the preferred direction (dashed line) of where a new anchor should be placed. Dots indicate targets and the star indicates the optimal new anchor position $\mathrm{x}^{+}$.

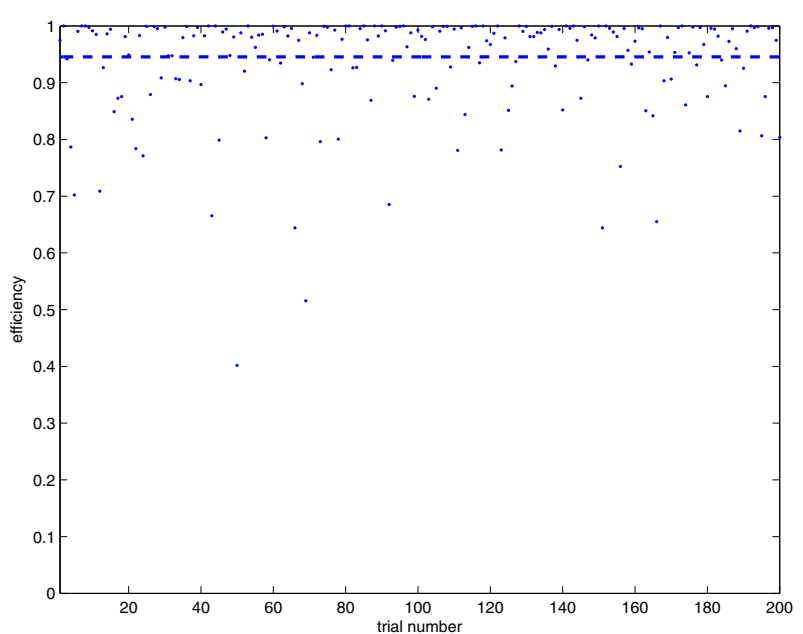

Fig. 5: Efficiency of anchor placement in 200 Simulation runs.

\section{CONCLusions}

In this paper we provide novel insights on the optimal anchor placement for wireless localization. For the single target scenario where all anchors can be placed freely it is shown that the directions of the anchors (as seen from the target) should form a tight frame. With this result, and relying on the large amount of properties known about tight frames, it is possible to generalize a number of previously known results while providing additional results and insights.

More specifically, we show that it is always possible to find optimal geometries for any number of anchors in both 2D and $3 \mathrm{D}$, and provide explicit formulas to select the anchor positions. Furthermore we show that every optimal geometry exhibits the property of maximum orthogonality between the directions of the anchors.

While these results are specifically for the single-target scenario, we also prove a result using frame theory that is useful for the anchor placement in multi-target networks, which has -in general- no closed-form solution. More specifically, we propose an intuitive low-complexity algorithm for anchor placement that sequentially minimizes the frame potential.

With an efficiency of $95 \%$, this algorithm can easily be used in systems with dynamic anchors where fast adaptation to a changing network topology is required.

\section{APPENDIX A \\ PROOF OF THEOREM 2}

Proof: Consider a set of range vectors $\left\{\mathbf{u}_{k}\right\}$ that form a frame with frame potential FP. Adding a new anchor can be formulated as adding a new range vector $\mathbf{u}^{+}$to the frame. Theorem 1 states that the necessary condition for this new anchor geometry to be optimal, is that the new frame is tight. From property 3 we know that a tight frame minimizes the frame potential such that we can formulate the problem as:

$$
\text { minimize } \mathrm{FP}_{\text {aug }}=\mathrm{FP}+\sum_{k}^{K}\left|\mathbf{u}_{k}^{\mathrm{T}} \mathbf{u}^{+}\right|^{2}
$$
It is also observed that some trials have a very low efficiency, for example trial 50 only has en efficiency of $e=0.4$. Closer inspection of the results showed that these outliers are the result of networks where the addition of a new anchor did not lower the CRLB much. As a result, a small discrepancy between the optimal CRLB and the obtained CRLB from the algorithm result is a low efficiency. with $\mathrm{FP}=\sum_{k, \ell}\left|\mathbf{u}_{k}^{\mathrm{T}} \mathbf{u}_{\ell}\right|^{2}$ the frame potential without the newly added anchor.

Notice that the original frame is related to a FIM $\mathbf{F}$ as follows: $\mathbf{F}=\sum_{k} \mathbf{u}_{k} \mathbf{u}_{k}^{\mathrm{T}}$. Let us consider the eigendecomposition of $\mathbf{F}=\mathbf{Q} \mathbf{\Lambda} \mathbf{Q}^{\mathrm{T}}=\sum_{i}^{M} \lambda_{i} \mathbf{q}_{i} \mathbf{q}_{i}^{\mathrm{T}}$, where $\boldsymbol{\Lambda}$ is a diagonal matrix 
with eigenvalues $\lambda_{i}$ and the corresponding eigenvectors $\mathbf{q}_{i}$ are the columns of $\mathbf{Q}$. We order the eigenvalues in ascending order, i.e. $\lambda_{1} \leq \lambda_{2} \leq \ldots \leq \lambda_{M}$ Hence, it follows that the original set of anchors is equivalent with a frame composed of the $M$ eigenvectors of $\mathbf{F}$ with the following equivalent frame potential:

$$
\mathrm{FP}_{\mathrm{aug}}=\mathrm{FP}+\sum_{i}^{M} \lambda_{i}\left|\mathbf{q}_{i}^{\mathrm{T}} \mathbf{u}^{+}\right|^{2}
$$

with $\mathrm{FP}=\sum_{k, \ell} \lambda_{k} \lambda_{\ell}\left|\mathbf{q}_{k}^{\mathrm{T}} \mathbf{q}_{\ell}\right|^{2}=\sum_{i}^{M} \lambda_{i}^{2}$, being the frame potential without the newly added anchor.

Because the eigenvectors of $\mathbf{F}$ span $\mathbb{R}^{M}$, we can write the range vector of the new anchor according to equation (4) as a linear combination of the eigenvectors of $\mathbf{F}$, that is, $\mathbf{u}^{+}=$ $\sqrt{F_{k}} \sum_{i}^{M} b_{i} \mathbf{q}_{i}$. Inserting this in (30) yields:

$$
\mathrm{FP}_{\mathrm{aug}}=\mathrm{FP}+F_{k} \sum_{i}^{M} \lambda_{i}^{2} b_{i}{ }^{2} .
$$

In order to minimize the augmented frame potential we need to minimize $\sum_{i}^{M} \lambda_{i}^{2} b_{i}{ }^{2}$ which is achieved when $b_{1}=1$ and $b_{i}=0$ for $\forall i \neq 1$. Or correspondingly, when the new anchor is in the direction of the minor eigenvector of $\mathbf{F}$, i.e, the eigenvector corresponding to the smallest eigenvalue.

\section{ACKNOWLEDGMENT}

This research is supported by the Belgian National Fund for Scientific Research (FWO Flanders).

\section{REFERENCES}

[1] N. Patwari, A. H. III, M. Perkins, N. S. Correal, and R. J. O'Dea, "Relative location estimation in wireless sensor networks," Signal Processing, IEEE Transactions on, vol. 51, no. 8, pp. 2137 - 2148, aug. 2003.

[2] B. Yang and J. Scheuing, "Cramer-Rao bound and optimum sensor array for source localization from time differences of arrival," in Acoustics, Speech, and Signal Processing, 2005. Proceedings.(ICASSP'05). IEEE International Conference on, vol. 4. IEEE, 2005, pp. iv-961.

[3] J. N. Ash and R. L. Moses, "On optimal anchor node placement in sensor localization by optimization of subspace principal angles," in Acoustics, Speech and Signal Processing, 2008. ICASSP 2008. IEEE International Conference on, 31 2008-april 4 2008, pp. 2289 - 2292.

[4] A. N. Bishop, B. Fidan, B. Anderson, K. Doğançay, and P. N. Pathirana, "Optimality analysis of sensor-target localization geometries," Automatica, vol. 46, no. 3, pp. 479 - 492, 2010.

[5] J. Kovacevic and A. Chebira, "Life beyond bases: The advent of frames (Part I)," Signal Processing Magazine, IEEE, vol. 24, no. 4, pp. 86-104, 2007.

[6] — "Life beyond bases: The advent of frames (Part II)," Signal Processing Magazine, IEEE, vol. 24, no. 5, pp. 115-125, 2007.

[7] S. M. Kay, Fundamentals of Statistical signal processing, Volume 2: Detection theory. Prentice Hall PTR, 1998.

[8] J. J. Benedetto and M. Fickus, "Finite normalized tight frames," Advances in Computational Mathematics, vol. 18, no. 2-4, pp. 357-385, 2003.

[9] P. Massey and M. Ruiz, "Tight frame completions with prescribed norms," arXiv preprint math/0606319, 2006.

[10] H. Wymeersch, J. Lien, and M. Z. Win, "Cooperative localization in wireless networks," In proc. of the IEEE, vol. 97, no. 2, pp. 427-450, Feb 2009. [Online]. Available: http://ieeexplore.ieee.org/lpdocs/epic03/wrapper.htm?arnumber=4802193

[11] P. Kułakowski, J. Vales-Alonso, E. Egea-López, W. Ludwin, and J. García-Haro, "Angle-of-arrival localization based on antenna arrays for wireless sensor networks," Computers \& Electrical Engineering, vol. 36, no. 6, pp. 1181-1186, 2010. 